\title{
Internet y Comunidad: Una aproximación desde el enfoque del estudio de redes personales
}

\author{
José Ignacio Porras Martínez \\ Universidad de Los Lagos, Santiago, Chile. \\ Email: joseiporras@yahoo.com
}

\begin{abstract}
Resumen: ${ }^{1}$ El propósito de este artículo es esencialmente aportar a la renovación metodológica en el campo de la investigación social. En concreto, se buscar presentar y fundamentar sobre las potencialidades del uso del estudio de las redes personales a partir de la aplicación de conceptos, métodos y técnicas propios del enfoque del Análisis de Redes Sociales (ARS). El método será desafiado como herramienta para abordar uno de los temas recurrentes en los debates sociológicos, la contigüidad espacial para la construcción de la comunidad. En concreto, nuestro soporte contextual será el aprendizaje obtenido a partir de la aplicación de este método en la investigación realizada para evaluar el impacto de Internet en la formación de capital individual y colectivo en un conjunto seleccionado de localidades de la Patagonia chilena caracterizadas por sus niveles críticos de aislamiento.
\end{abstract}

Palabras clave: análisis de redes sociales, redes personales, metodología, comunidad, internet.

\section{Internet and Community: An approach in the perspective of the study of personal networks}

\begin{abstract}
The aim of this paper is to make a contribution to the renewal of social research. Specifically, we seek to provide a state-of-the-art about the personal networks studies from the application of concepts, methods and techniques of Social Network Analysis (SNA). The method will be challenged as tool to approach one of the most relevant issues of modern sociology, the spatial conditions for community building. The framework support will be the learning obtained from the application of this method in the research undertook to evaluate the impact of Internet in the individual and collective social capital formation in a selected group of Chilean Patagonia's locality, characterized by its critic levels of isolation.

Key words: social networks analysis, personal networks, methodology, community, internet

\section{Internet e Comunidade: Uma abordagem desde o enfoque do estudo das redes pessoais}

Resumo: O objetivo deste artigo é, essencialmente, contribuir para a renovação metodológica no campo da pesquisa social. Concretamente, se procura apresentar fundamentar o potencial do uso do estudo das redes pessoais a partir da aplicação de conceitos, métodos e técnicas próprios do enfoque da Análise de 
Redes Sociais (ARS). O método será desafiado como uma ferramenta para abordar um dos temas recorrentes em debates sociológicos, a contiguidade espacial para a construção da comunidade. Especificamente, o nosso apoio contextual será a aprendizagem obtida a partir da aplicação deste método na pesquisa realizada para avaliar o impacto da Internet sobre a formação de capital individual e coletivo em um conjunto selecionado de localizações na Patagônia chilena, caracterizada por seus níveis críticos de isolamento.

Palavras-chave: análise de redes sociais, redes pessoais, metodologia, comunidade, internet.

$* * *$

\section{Introducción}

A pesar de la importancia que desde los inicios de la sociología ha tenido el concepto de comunidad, todavía permanece como uno de los más complejos de definir y, por tanto, de operativizar y estudiar (Cohen, 1985). A ello contribuye, sin duda, las pasiones que ha generado en los debates abiertos en la sociología moderna. Entre ellos, uno en particular concentra nuestra atención en este artículo. Se trata de la discusión sobre la disolución de la idea de comunidad enfrentada a la modernidad. A partir de los trabajos de autores clásicos como Tönnies, Durkheim, Simmel o Weber, esta línea de trabajo se plantea con mayor o menor intensidad la progresiva destrucción de las formas tradicionales de asociación frente a los procesos de urbanización e industrialización que comenzaron a desarrollarse a escala global en el tránsito del siglo XIX al XX (Sampson, 1999), llegando incluso hasta nuestros días a manos de autores como el D. Putnam (1996 y 2002) o Bauman (2003).

La comunidad se ha perdido y se ha redescubierto muchas veces a lo largo del tiempo en que se ha desarrollado este debate, de forma que es necesario cierta precaución para no balancear entre las posturas que nos llevan a una mirada nostálgica de la idea de comunidad o a su total desaparición con el paso del tiempo. Una apuesta más razonable sugiere avanzar hacia la comprensión de los cambios que los procesos comunitarios han sufrido durante las últimas décadas en un contexto general marcado por profundas transformaciones. Para ello es necesario renovar las herramientas metodológicas que dispone el investigador para poder afrontar con mayor certeza de éxito los desafíos que plantea la revisión conceptual sobre la comunidad. El propósito de este artículo se verá cumplido si contribuye a este objetivo general.

Para ello nos proponemos presentar y fundamentar las potencialidades de la aplicación de las herramientas que provee el enfoque metodológico del Análisis de Redes Sociales (ARS) para explicar el requisito de la contigüidad espacial para la construcción de la comunidad. Más concretamente, el método centrado en el estudio de redes personales. Nuestra propuesta se asienta en los resultados y el aprendizaje de la aplicación de esta metodolo- 
gía en la investigación realizada para evaluar el impacto del uso de Internet en el capital social individual y colectivo de aquellas localidades de la Patagonia chilena caracterizadas por sus niveles críticos de aislamiento.

El punto de partida de nuestra aproximación será discutir brevemente la impronta que las nuevas tecnologías de la información y comunicación $\mathrm{y}$, en particular, Internet influyen en la variable espacial sobre la que tradicionalmente se ha fundado la idea de comunidad. A partir del establecimiento de este primer marco de referencia, pasaremos a fundamentar aquellos elementos del estudio de redes personales que justifican la potencialidad para abordar el desafío propuesto, estudiar las consecuencias de la translocalización de las relaciones sociales como consecuencia del uso de Internet. En un tercer apartado, presentaremos la investigación, sus principales hallazgos y, principalmente, la estrategia seguida para alcanzarlos. Por último, en el cierre del artículo, se realizará una reflexión general que considere las fortalezas y debilidades de la propuesta planteada.

\section{La comunidad: de la contigüidad espacial a la construcción del "nosotros"}

La idea de comunidad fue durante mucho tiempo entendida como un derivada de la interacción basada en la proximidad geográfica (Hillery, 1955). Una “unidad territorial” (Poplin, 1972), definida por límites físicos que encierran un espacio geográfico compartido donde se construían, casi de forma obligada, una densa red de vínculos primarios. El componente topográfico asociado al concepto de comunidad empezó a ser cuestionado en la medida que el desarrollo científico-técnico empezó a diluir las distancias espaciales y temporales, mediante el desarrollo de los medios de transporte y especialmente las tecnologías de la información y comunicación (Wellman, 1998; Fischer, 1982, 1994). Ahora bien, lo que se plantea no es que nos encontremos en la antesala del final de la vida comunitaria como consecuencia de estas transformaciones, sino de su progresiva transformación como consecuencia del final de las pautas de sociabilidad. Tal y como plantea Wellman (2011), desde el «puerta a puerta», el «lugar a lugar»y, finalmente, el "persona a persona», haciendo compatibles y no excluyentes diferentes formas de comunidad. Por su parte, Lee y Newby (1983) afirma que las personas que viven próximas no implica necesariamente que tengan relación alguna, de forma que, más que deteriorarse y desaparecer, la actividad social se ha liberado progresivamente de la necesidad de anclaje físico, poniendo como base la elección por afinidad más allá de la simple contigüidad (Wellman y Wellman, 1992). Un argumento que se asocia directamente con el concepto de homofilia, introducido en la teoría social por Lazarsfeld y Merton (1955). La homofilia, del griego “amor a los iguales”, identifica la tendencia de las personas de relacionarse con personas que se parecen a ellas. Más formalmente, si dos personas tienen atributos similares en mayor número que el resto de la población tendrán mayor probabilidad de relacionarse entre ellos que con los demás (Verbrugge, 1977). Al respecto de los 
atributos que propician esta relación entre iguales, Lazarsfeld y Merton distinguen entre aquellos que son inherentes (sexo, raza, edad,..) y aquellos que son adquiridos (educación, status social, aficiones, creencias,...). La discusión se plantea, al referirse a estos últimos, si las causas de la homofilia operan desde aquellos que se buscan por compartir determinados atributos o desde una estructura social dada. Por ejemplo, cabe preguntarse si el hecho de que los estudiantes de una determinada institución académica compartan determinados hábitos sociales (forma de vestir, aficiones, expectativas,...) es consecuencia o no de estar juntos en ese espacio social. Desde esta perspectiva, las investigaciones sobre homofilia tienden a concentrarse en identificar aquellas condiciones bajo los cuáles es más probable que opere este principio (McPherson, Smith-Lovin y Cook, 2001), abriendo el interrogante sobre el impacto que ha tenido Internet en propiciar nuevas pautas de sociabilidad que alteren los presupuestos tradicionales de la vida comunitaria.

A pesar de que en las últimas décadas han crecido exponencialmente las investigaciones que relacionan Internet con las pautas de sociabilidad (Antoci, Sabatini, y Sodini, 2012; Bauernschuster, Falck, y Woessmann, 2011; Beaudoin, 2008; Burke, Kraut, y Marlow, 2011; Ellison, Steinfield, y Lampe, 2007; Kraut et al., 2002; Miyata y Kobayashi, 2008; Pénard y Poussing, 2010; Sum, Mathews, Pourghasem, y Hughes, 2008; Valenzuela, Park, y Kee, 2009),, estamos lejos todavía de un consenso sobre sus consecuencias. Hay estudios que concluyen que el uso de Internet aísla a los individuos y reduce el tiempo que gastan en actividades sociales cara-acara y cívicas (Putnam, 1995). Pero también hay fuertes evidencias de que su uso puede fortalecer vínculos o crear nuevos lazos que complementan a los ya existentes (ver Kraut et al. 2002; Lin, 2001; Pénard y Poussing, 2010).

La evidencia acumulada durante los últimos años, principalmente en el contexto de la aparición de las aplicaciones asociadas a la Web 2.0, indican que las herramientas disponibles en Internet permiten la creación de interacciones sociales entre personas con intereses, gustos e inquietudes similares y que se relacionan para intercambiar información, pero también para construir conocimientos colectivos (Wellman, 2001; Miyata y Kobayashi, 2008; Prenard, 2012; Lin, 2001). Pero existe otro elemento en la construcción de comunidad que transciende los vínculos simples o múltiples que pueden establecer un conjunto de personas o actores en función de percibirse mutuamente dependiente. Nos referimos al elemento identitario. Como plantea Sennett "una comunidad es una particular variedad de un grupo social en la que los hombres [y mujeres] creen que comparten algo juntos. [...] El vínculo de comunidad es el de percibir identidad común, un placer en reconocernos a nosotros y a lo que somos” (Sennet, 2001: 71).

Internet juega un papel importante en la creación de todas clases de identidades. Una parte importante de la literatura sobre el impacto de Internet en la construcción de identidades se ha centrado en las identidades personales. Al respecto, autores como Giddens (1991), Turkle (1995) o Bell (2001) coinciden en señalar que la emergencia de las nuevas tecnologías de la 
información y comunicación, incluido Internet, han permitido desencadenar un proceso hacia una identidad que deja atrás la esencialidad basada en los condicionantes temporales o espaciales para avanzar hacia una concepción de la misma como un constructo, movible y múltiple. Desde esta línea argumentativa, y al retomar el foco de nuestro artículo, resulta interesante observar como Internet modifica el soporte territorial de la identidad comunitaria en dos direcciones contradictorias. En algunos casos, tal y como se ha señalado previamente, la emergencia de comunidades virtuales que se forman a partir de intereses compartidos entre personas localizadas en los más diversos puntos de la geografía marginan hacia la mayor irrelevancia la variable territorial en la construcción identitaria. En otros, sin embargo, Internet es el medio que permite preservar las identidades enraizadas en un determinado territorio. Son varias las investigaciones que han estudiado las llamadas comunidades virtuales de la diáspora que convocan a una especie de "patriotismo digital" a personas que, independientemente de su localización, se perciben como parte de una determinada identidad nacional, regional o local. En otras palabras, si a un lado siempre fue posible al individuo dislocarse de su región/país de origen a otro, Internet también permite este mismo "movimiento" sin que sea necesario tal dislocamiento físico, mas solamente virtual, así como permite un rencuentro, y una especie de "retorno" a los orígenes (Tavskko, 2013).

Se trata, por veces, de un retorno de la diáspora a su tierra y cultura originales - comprendida por ancestral o anterior - sin la necesidad de cualquier tipo de desplazamiento físico, donde exista la posibilidad de compartir, teniendo por base signos, mitos y símbolos que identifican a todos con una comunidad. Y esta es más que una comunidad de destino fija, inmutable, presa al origen étnico/nacional, es una comunidad que migra, una identidad que migra (Bauman, 2004).

\section{El método de estudio de las redes sociales personales}

\section{Introducción al Análisis de Redes Sociales}

Durante las últimas décadas hemos sido testigos de amplio desarrollo del Análisis de Redes Sociales (Social Network Analysis en inglés). Una aproximación al estudio de las estructuras sociales que ha alcanzado altos niveles de sofisticación metodológica y técnica. Desde la variedad de formas de concebir el Análisis de Redes Sociales (ARS) resulta posible extraer una primera definición compartida que lo identifica como el estudio formal de las relaciones entre actores y de las estructuras sociales que surgen de la recurrencia de esas relaciones. La unidad básica de análisis del ARS es, por tanto, el dato relacional o vínculo específico existente entre un par de elementos (Wasserman, 1994). El dato relacional surge de la comunicación, colaboración, transacción, valoración, etc...existente entre un número determinado de "nodos", sean éstos organizaciones o personas. A partir de estos datos y de su procesamiento es posible construir la existencia de las redes sociales que vinculan directa e indirectamente a todos los nodos. 
Detrás de este interés por relevar la existencia de redes sociales se encuentra el convencimiento de que más allá de las prescripciones normativas, los atributos personales o las relaciones diádicas o bilaterales, las redes facilitan o restringen el acceso de las organizaciones o personas a recursos tales como la información, la riqueza o el poder (Granovetter, 1973; Scott, 1992; Wasserman y Faust, 1994; Watts, 1999, Wellman, 2000; Barabasi, 2003;). El conocimiento de las propiedades estructurales que caracterizan a las redes sociales, por tanto, nos pueden ayudar a comprender, predecir e incluso gestionar mejor los resultados de la acción humana. Así, por ejemplo, la densidad o proporción de relaciones existentes entre los nodos de una red sobre el total de relaciones posibles es indicador del grado de intensidad de los vínculos. Por otro lado, conocer la forma en que los distintos nodos se conectan a la red o su ubicación al interior de ella nos ofrece nuevas variables para comprender su poder de intercambio con sus pares y también su comportamiento. La intuición estructural nos lleva a considerar que un actor que se encuentre en una posición intermedia en el camino más corto entre dos actores ejercerá su influencia sobre éstos.

La estructura de relaciones sociales alrededor de una persona, grupo u organización no sólo es causa de su comportamiento, sino que también es su resultado. El ARS va más allá de considerar la estructura de relaciones entre actores y el posicionamiento del actor en la red como características con consecuencias importantes para el comportamiento, percepción y actitud de las unidades individuales y el sistema de conjunto (Knoke y Kuklinski, 1982). Por tanto, propone estudiar las estructuras sociales de forma directa y concreta. Ello implica analizar las estructuras ordenadas de relaciones establecidas entre los miembros del sistema social. Y con ello elaborar un mapa de dichas estructuras, describir sus pautas e intentar descubrir los efectos de las pautas relacionales en el comportamiento de los individuos miembros de dicha estructura social.

\section{La aproximación egocéntrica en el Análisis de Redes Sociales}

Existen dos aproximaciones distintas desde las que se aproxima el ARS a comprender las estructuras sociales. Una primera aproximación es la que aporta explicaciones sobre las propiedades generales de la estructura de relaciones entre un grupo de actores definido. Es lo que se conoce en la literatura del ARS como la aproximación sociocéntrica y cuyo principal precursor fue Jacob Moreno (1934) con el desarrollo de la sociometría. Una segunda aproximación a la red social tiene su foco en un determinado individuo o actor, ego en la nomenclatura del ARS, y busca reconstruir la estructura de relaciones que se construyen a partir de él tomando en consideración los otros individuos o actores, los llamados alteri, tomando en considerando tanto las relaciones entre éstos y ego como las relaciones entre ellos. Será la combinación entre estas dos aproximaciones las que permite avanzar en una de las líneas de trabajo teórico más promisorias que aporta el ARS, la superación de la tradicional dualidad entre enfoque macro y micro concentrándose en un nivel meso del análisis. El supuesto que sustenta esta promesa explicativa que contribuye a construir el ARS es la concep- 
ción de la realidad social como una red de redes conformando por la integración de actores y vínculos, tanto en términos de personas como de organizaciones, en diferentes niveles. La propuesta planteada en este artículo tiene su eje en la aproximación a la estructura de relaciones sociales desde la mirada egocéntrica o el estudio de redes personales, entendiendo que desde este punto de partida y tras combinarse con la aproximación sociocéntrica o completa a la red estaremos en mejores condiciones para la comprensión de fenómenos sociales de rango intermedio o meso (Ferrand, 2002; de Federico, 2002).

La mirada egocéntrica tiene su primer referente en Elisabeth Bott (1955). Esta autora, al igual que el resto de antropólogos urbanos de la Escuela de Manchester, encontró en el ARS un complementó al paradigma estructural-funcionalista dominante en aquella época (Molina, 2005). Dado el dinamismo y la fluidez de la realidad de las ciudades, este paradigma, que asumía la existencia de una red activa de instituciones que permitían dar cuenta de la vida social, aparecía como insuficiente para poder explicar el comportamiento de los individuos. Bajo estas circunstancias, el estudio referido de E. Bott se centró en las unidades domésticas de las parejas que vivían en las grandes ciudades inglesas para establecer la correlación existente entre el grado de segregación de los roles conyugales y la densidad de las redes sociales.

Una segunda derivada de este enfoque de trabajo iniciado por la Escuela de Manchester se enlaza directamente con el tema de nuestro interés, la vida comunitaria. Autores como Laumann (1973), Wellman $(1979,1982,1988)$, Fisher (1982) o Ferrand (1999) aplican las herramientas propias del estudio de las redes egocéntricas o las redes personales para levantar una amplia cantidad y variedad de evidencia empírica, muchas veces proporcionada por estudios longitudinales, con la que poner en cuestión la preponderancia de los factores territoriales en la construcción de las relaciones sociales. Esta forma de aproximarse a los estudios de comunidad, que tomo mayor fuerza tras la instalación de Internet en la vida cotidiana de las personas, propició tanto su desarrollo metodológico como su sofisticación técnica. Gran parte del desarrollo metodológico vino dado por la capacidad de perfeccionar la forma de identificar los llamados alteri o contactos a partir del diseño de encuesta con generadores de nombres. Se trata de preguntas que permiten identificar relaciones en los más variados escenarios sociales en función de los intereses del investigador (domésticos, profesionales, ocio, emergencias,...). Algunos ejemplos conocidos de este tipo de preguntas serian los siguientes:

1. Si tuviera que ausentarse por un motivo inesperado, ¿̇a quién(es) confiaría el cuidado de sus hijos pequeños?

2. Si tuviese que pedir prestado una cantidad importante de dinero, ¿a quién lo haría?

3. En los últimos tres meses ¿con quién(es) ha compartido alguna actividad de ocio después del trabajo o durante el fin de semana? 
Estrechamente vinculado a esta literatura cabe situar aquella derivada del famoso experimento desarrollado por Stanley Milgram (1967) conocido como el Mundo Pequeño (Watts, 1999; Barabási, 2002)². El intento por estimar el número de contactos promedio de una persona y, principalmente, la estructura de relaciones derivada de estos contactos ha desafiado a los analistas de redes a repensar las herramientas metodológicas aplicadas. Dentro de este contexto, uno de los aportes más significativos se encuentra en la propuesta planteada por Christopher McCarty. Su punto de partida es que sólo en la medida en que el estudio de redes personales integre la aproximación sociocéntrica o de red completa puede ser entendido propiamente como un análisis estructural de redes. De lo contrario, lo que se hace es describir las redes utilizando medidas que no son estructurales, recurriendo en su lugar a un análisis de base-atributiva que resume las relaciones de los encuestados con los miembros de la red (McCarthy, 2010). Para operar esta integración entre la mirada egocéntrica y sociocéntrica propone el autor la aplicación de una encuesta en la que, en primer lugar, los encuestados identifican a sus contactos y, en segundo lugar, establecen las relaciones significativas existentes entre ellos. Un instrumento bastante largo y tedioso para el encuestado, pero cuyos datos obtenidos resultan muy reveladores de los condicionantes estructurales en que se encuentran las personas insertos en su red personal.

Por último, una tercera línea de trabajo que ha propiciado en los últimos años nuevos aportes al estudio de las redes personales se encuentra en los estudios sobre el capital social individual. Un referente destacado dentro de este ámbito es Nan Lin (1999), quién identifica la fuente del capital social en la interacción entre individuos y los recursos “circulantes" en sus relaciones. Lo que plantea una concepción meso del nivel de análisis. Para poder medir de forma adecuada el capital social definido de esta forma se hace necesario conocer las posiciones sociales de los alteri por una parte y el nivel de acceso existente en el entorno por la otra (Molina, 2005). A partir de este propósito, modificó la práctica común seguida hasta el momento en los estudios de redes personales de concentrar los esfuerzos en indagar sobre nombres de contactos para pasar a indagar sobre posiciones sociales. El supuesto es que a partir de una muestra conveniente de las profesiones y posiciones existentes en una estructura social dada y preguntando el número de personas conocidas en cada una de las posiciones, es posible obtener una aproximación al capital social potencialmente accesible (Lin 2001 citado en Molina, 2005). El tipo de preguntas generadora de nombres que plantea Lin en su propuesta son, una vez definida una muestra convincente de profesionales y posicionales, las siguientes:

1. ¿Conoce a alguien que tenga este empleo?

2. ¿Cuánto hace que la conoce?

3. ¿Cuál es su relación con ella?

4. ¿Podría encontrar a una persona así a través de algún conocido?

El resultado esperado son datos que nos permitan obtener una aproximación al capital social potencialmente accesible. Asimismo, y con tenien- 
do información sobre la intensión de la relación, se podría predecir el capital social movilizable en un momento dado por una acción intencional de ego.

\section{Desafíos para el desarrollo del estudio de redes personales}

El desarrollo de estudios personales presenta problemas que siguen desafiando en la actualidad la capacidad innovadora de los metodólogos en la comunidad del ARS. El principal problema se encuentra asociado a la fiabilidad de los datos obtenidos a partir de la aplicación de encuestas. Si la memoria siempre es selectiva en cualquier tipo de investigación, en el caso de los estudios de redes mucho más. El motivo es doble. En primer lugar porque existe una tendencia generalizada a vernos más centrales de lo que realmente somos cuando informamos de nuestras relaciones y de las relaciones de los demás. Tal y como plantea Burt (1994), por ejemplo, cuando ego informa las relaciones de sus alteri tiende a conectarlos a través suyo. Asimismo, también tendemos a enfatizar aquella relaciones que tenemos con las personas que ocupan un estatus similar o superior a nosotros, olvidando los que tienen un estatus inferior (Molina, 2005). Por otro lado, el sesgo existente en la entrega de la información por parte del encuestado tiende a potenciarse tanto por la extensión del instrumento como por lo tedioso de las preguntas. Si tomamos, por ejemplo, la propuesta original de McCarthy de considerar un mínimo de 40 personas como base para poder levantar una red personal que transcienda los vínculos fuertes (familia, amigos íntimos,..) e incorpore aquellos que podemos considerar como débiles (compañeros de universidad, del trabajo, conocidos,..) estaremos, al momento de completar la matriz de relaciones entre alteri, trabajando con un instrumento de 780 preguntas.

Existen algunas recetas para tratar de moderar el impacto de estos problemas. Una de las más socorridas es considerar la aplicación de instrumentos cualitativos que permitan completar y validar la información obtenida a través de la encuesta ${ }^{3}$. Otra alternativa, como parte del propio diseño de la encuesta, seria limitar la cantidad de preguntas concentrando la atención en un número más reducido de contactos o alteri, así como en vínculos a largo plazo o donde se produzca una mayor intensidad en las relaciones. Lo que permite obtener mayor nivel de fiabilidad en los datos. Por último mencionar el apoyo que supone el desarrollo de determinados software, expresamente desarrollados para trabajar el estudio de redes personales, que facilitan la toma de información. Uno de los más conocidos es el software desarrollado por el propio Christopher McCarthy, Egonet ${ }^{4}$.

\section{Caso de estudio: Internet y Capital Social en zonas aisladas}

\section{Aspectos generales de la investigación}

La investigación que nos sirve como marco de nuestra propuesta metodológica tuvo como objetivo principal evaluar el impacto de Internet en la formación y acceso al capital social en comunidades con niveles críti- 
cos de aislamiento geográfico en la Patagonia chilena ${ }^{5}$. En la línea de los estudios que relacionan Internet y Capital Social mencionados en la primera parte de este artículo, se trataba de validar un conjunto de hipótesis de trabajo que planteaban tentativamente que el acceso y uso regular de Internet modifica el patrón de relaciones sociales de las personas al ampliar y modificar el número de vínculos y, por tanto, generando mayores oportunidades para el acceso y la movilización de recursos. El elemento más novedoso de nuestra investigación con respecto a los estudios señalados fue la incorporación de la dimensión del aislamiento territorial. Al incorporar esta dimensión no sólo se buscaba abordar un escenario que apenas había sido tratado dentro de este tipo de investigaciones, sino también encontrar un escenario más propicio para identificar los mecanismos a través de los cuáles el stock de capital social obtenido por las personas a través de Internet se transfería o no hacia el stock de capital social acumulado por la comunidad en su conjunto. El supuesto que sustentaba estaba posibilidad era que en aquellas localidades que presentaban niveles críticos de aislamiento se produce un fenómeno de clausura relacional en términos del peso de las limitantes geográficas en la formación de vínculos sociales, alta densidad de relaciones entre sus habitantes y circulación de recursos redundantes. Bajo estas circunstancias cabía suponer que Internet abriera canales alternativos de obtención de recursos externos por parte de la comunidad a través de la función mediadora de sus usuarios más ávidos y capaces para aprovechar sus potencialidades. Es lo que identificamos como "puente interlocal digital”. Al poco de iniciarse la investigación, la eclosión de un movimiento regionalista en la región de Aysén y el uso de Internet como herramienta para la acción colectiva reforzó nuestro interés por identificar la emergencia de este tipo de intermediarios.

La investigación tuvo un carácter exploratorio. El diseño metodológico correspondió a un estudio de casos correspondiente a seis comunas de la región de Aysén seleccionadas en razón de ser categorizadas por un nivel de aislamiento crítico según fuentes oficiales (nota). Adicionalmente se sumaron dos comunas de la región Metropolitana, identificadas por las mismas fuentes con un nivel de aislamiento alto. Su inclusión se justifica por el interés de poder comparar los datos obtenidos en los casos de la región de Aysén con una realidad que si bien comparte la condición de aislamiento, la cercanía al principal polo urbano de Chile marca diferencias sustanciales al considerar esta variable territorial al evaluar el impacto de Internet en el capital social de sus habitantes.

El principal instrumento utilizado en la investigación fue una encuesta aplicada a un universo compuesto por personas mayores de 14 años que residen en las localidades seleccionadas por su grado de aislamiento y que cumplían la condición de ser usuarios habituales de Internet. Los casos fueron seleccionados en un muestreo de carácter aleatorio simple por cuotas por localidad. La aplicación de la encuesta fue presencial y abarca 623 casos entre septiembre y diciembre de 2012. Con anterioridad a la aplicación de la encuesta, y como uno de los insumos principales para su diseño, se realizaron 20 entrevistas semi-estructuradas a informantes claves en todas 
las comunas consideradas (autoridades locales, jefaturas de los principales departamentos en el municipio, jefaturas en servicios públicos, líderes sociales,...). Asimismo, en la etapa posterior a la aplicación de la encuesta, se desarrollaron un total de 8 entrevistas grupales a un grupo seleccionado de los propios encuestados para completar y validar los datos obtenidos.

\section{Diseño y aplicación del estudio de redes personales a la investigación}

Dentro de este diseño metodológico, la incorporación de un estudio de redes sociales a una submuestra de 22 casos dentro de la muestra general de la encuesta tenía como propósito ahondar, principalmente, sobre la transición del capital social individual al colectivo. Los casos fueron seleccionados siguiendo el supuesto del diseño muestral estructural. Esto es, representativas desde la perspectiva de que "cada miembro seleccionado representa un nivel diferenciado que ocupa en la estructura social del objeto de investigación” (Mejias, 2000, pág. :105).

\section{Tabla 1. Muestra de casos}

\begin{tabular}{|c|c|c|c|c|c|}
\hline Ego & $\begin{array}{c}\text { Lugar de } \\
\text { Residencia }\end{array}$ & $\begin{array}{c}\text { Uso } \\
\text { Internet }\end{array}$ & Edad & $\begin{array}{c}\text { Nivel de } \\
\text { Escolaridad }\end{array}$ & Actividad Principal \\
\hline Emilia & $\begin{array}{c}\text { Puerto } \\
\text { Tranquilo }\end{array}$ & Alta & 30 & $\begin{array}{l}\text { Estudios de } \\
\text { Postgrado }\end{array}$ & Antropóloga \\
\hline Marcela & $\begin{array}{c}\text { Puerto } \\
\text { Tranquilo }\end{array}$ & Alta & 42 & $\begin{array}{l}\text { Enseñanza Media } \\
\text { Incompleta }\end{array}$ & Servicio turistico \\
\hline Victoria & $\begin{array}{c}\text { Puerto } \\
\text { Tranquilo }\end{array}$ & Alta & 38 & Enseñanza Media & Locutora radial \\
\hline Blanca & $\begin{array}{c}\text { Puerto } \\
\text { Tranquilo }\end{array}$ & Alta & 49 & $\begin{array}{l}\text { Enseñanza Media } \\
\text { Incompleta }\end{array}$ & Locutora radial \\
\hline Andrés & $\begin{array}{c}\text { Puerto } \\
\text { Tranquilo }\end{array}$ & Alta & 33 & Universitario & Funcionario público \\
\hline Eduardo & Tortel & Baja & 48 & Enseñanza Media & Ganaderia \\
\hline Gabriela & $\begin{array}{l}\text { Cerro } \\
\text { Castillo }\end{array}$ & Baja & 31 & Nivel Técnico & Dueña de casa \\
\hline Teledofis & $\begin{array}{l}\text { Cerro } \\
\text { Castillo }\end{array}$ & Baja & 69 & Enseñanza Media & $\begin{array}{l}\text { Comerciante (artesanis - } \\
\text { hospedaje) }\end{array}$ \\
\hline Silvia & $\begin{array}{c}\text { Villa } \\
\text { O'Higgins }\end{array}$ & Baja & 21 & $\begin{array}{l}\text { Universitaria } \\
\text { completa }\end{array}$ & Turismo \\
\hline Manuel & $\begin{array}{c}\text { Puerto } \\
\text { Tranquilo }\end{array}$ & Baja & 76 & Enseñanza Media & Agro Turismo/Ganaderia \\
\hline Ciro & O Higgins & Alta & 59 & Enseñanza Media & Construcción \\
\hline Ernel & Tortel & Alta & 45 & Enseñanza Media & Encargado de biblioteca \\
\hline Juan & Tortel & Alta & 33 & Enseñanza Media & Trabaja independiente \\
\hline Susana & Tortel & Alta & 28 & Nivel Técnico & Funcionaria municipal \\
\hline Daniel & O Higgins & Alta & 43 & Universitario & Turismo \\
\hline Fernanda & O Higgins & Alta & 16 & Enseñanza Básica & Estudiante \\
\hline Marcela & O Higgins & Alta & 28 & Enseñanza Media & Comerciante \\
\hline Verónica & O Higgins & Alta & 32 & Enseñanza Media & Encargada de biblioteca \\
\hline Mirns & O Higgins & Alta & 32 & Enseñanza Media & Secretaria administrativa \\
\hline Maria José & $\begin{array}{l}\text { Cerro } \\
\text { Castillo }\end{array}$ & Alta & 17 & Enseñanza Básica & Estudiante \\
\hline
\end{tabular}

El diseño del instrumento aplicado responde, básicamente, al método propuesto por Christopher McCarthy (Ibid) para el estudio de redes personales. Esto es, combinar la aproximación sociocéntrica de la estructura de relaciones formada por ego con su identificación a partir de un conjun- 
to de preguntas sobre variables atributivas referidas a sus relaciones dentro de esta red. Tal y como se expone en la tabla n.2, en su primera parte la encuesta se concentró en caracterizar a ego en función de algunas variables sociodemográficas y su uso de Internet. Posteriormente la atención se concentró en la identificación de alteri. La pregunta utilizada parar generar nombres fue la siguiente: “Si tuviera algún problema de carácter económico, de salud, del trabajo,... ¿a quién le pediría de apoyo?’. La formulación de esta pregunta tenía como objetivo circunscribir el objeto de análisis no a la generalidad de contactos de ego, sino solamente a aquellos alteri que fueron percibidos por éste como una fuente de apoyo efectivo. Posteriormente cada uno de los alteri fueron identificados por el encuestado en función de su categoría, tipo de apoyo esperado, lugar de residencia y principal medio de comunicación. Por último, la tercera parte de la encuesta tenía como propósito levantar la matriz de la estructura de relaciones entre los alteri identificados por ego. En concreto, la pregunta aplicada fue: "Entendiendo relación como la comunicación presencial o a distancia durante los últimos 3 años, ¿tiene usted conocimiento de que A (nombre del primer alteri) se relaciona con B (nombre del segundo alteri)?’. Esta pregunta fue aplicada de forma sucesiva hasta completar la totalidad de los alteri. Dado que por el tipo de dato y la fuente única de información la relación entre los distintos alteri siempre iba a ser recíproca (si A se relaciona con B, B se relaciona con A) la matriz resultante cumplía la condición de simetría y con vínculos no orientados.

Tabla 2. Variables en el Instrumento.

\begin{tabular}{|c|c|}
\hline Variables Atributivas Ego & Variables Atributivas Alteri \\
\hline Edad & $\begin{array}{c}\text { Categoría } \\
\text { (Familia, amigo, } \\
\text { compañero de trabajo y vecino) }\end{array}$ \\
\hline Nivel de escolaridad & $\begin{array}{c}\text { Actor al que se recurre en caso de } \\
\text { problemas o necesidad de apoyo } \\
\text { (Económico, Salud, Trabajo, Estudios, } \\
\text { Ocio) }\end{array}$ \\
\hline Ocupación principal & $\begin{array}{c}\text { Lugar de residencia } \\
\text { (Comuna, región, otras regiones, } \\
\text { fuera del pais) }\end{array}$ \\
\hline Lugar de uso de Intemet & $\begin{array}{c}\text { Tipo de contacto } \\
\text { (Presencial, Telefónico, Intemet, Otros) }\end{array}$ \\
\hline Frecuencia de uso de Intemet & \\
\hline $\begin{array}{c}\text { Frecuencia de uso de Intemet para } \\
\text { relacionarse } \\
\text { con familiares o personas cercanas }\end{array}$ & \\
\hline $\begin{array}{c}\text { Frecuencia de uso de Intemet para } \\
\text { buscar establecer con conocidos o } \\
\text { nuevas personas }\end{array}$ & \\
\hline
\end{tabular}

La encuesta fue aplicada de forma presencial por parte de los propios miembros del equipo del proyecto de investigación en los lugares 
definidos por los encuestados durante el mes de mayo 2013. Cinco meses después de la aplicación de la encuesta general. Con anterioridad a la aplicación del instrumento a testearlo en dos casos identificados como representativos del conjunto, lo que permitió realizar algunos ajustes menores en la formulación de las preguntas y disponer de la validación final del documento. La aplicación del instrumento estuvo precedida de la entrega de información sobre la investigación y objetivos, así como la presentación de la carta de consentimiento informado. No hubo rechazo. Todos los casos seleccionados previamente aceptaron responder la encuesta de redes.

El tiempo de aplicación de la encuesta fue variado en razón del tipo de encuestado y la cantidad de alteri mencionados en cada caso. Sin embargo, es posible establecer un promedio de alrededor de 40 minutos considerando la presentación del instrumento y el cierre final. Los principales problemas en la aplicación del instrumento que pudieran poner en cuestión la fiabilidad de los datos se concentraron en dos aspectos. En primer lugar, en la segunda parte del instrumento las dificultades de los encuestados para discernir entre la principal categoría de los alteri mencionados (ej: más amigo que vecino), el tipo de apoyo recibido (ej: problema de salud o económico) o el medio de contacto (ej: presencial o Internet). El segundo lugar, y al momento de levantar la matriz, la confusión que en algunas ocasiones provoca el tedio de las preguntas repetitivas sobre las relaciones entre los alteri. Conociendo de antemano esta posibilidad, la prevención para evitar esta situación fue orientar de forma constante al encuestado sobre el sentido de las preguntas.

El plan de análisis consideró el cálculo de las siguientes propiedades estructurales de red: densidad o porcentaje de vínculos existentes con respecto a los vínculos efectivos en la red; cliques o subestructura existentes formada por una triada de actores o mas; centralidad o posicionamiento de un actor en la red en función de sus vínculos directos con el resto de actores (centralidad de grado), acceso directo o indirecto al resto de actores (centralidad de cercanía) o posición intermediaria en la conexión indirecta entre dos actores (centralidad de intermediación).

El número final de casos considerados fue de 20, dado que se retiraron dos encuestas porque no contenían un número mínimo de alteri para que tuvieran valor analítico. En términos metodológicos, se realizaron cruces entre las matrices de relaciones derivadas de los 20 cuestionarios seleccionados y se analizaron gráficamente con los atributos de uso de Internet y lugar de residencia de los nodos componentes de cada una de las redes. Luego se extrajeron medidas de centralización de red (grado, intermediación y cercanía) y densidad. Del mismo modo, se exploró la existencia de subgrupos dentro de la red (cliques) y adicionalmente se estudió un indicador (E Index) que permitió verificar la orientación (local o extralocal) de cada una de las redes. Para estos efectos, y con la finalidad de facilitar el posterior análisis estadístico, los indicadores de redes y atributos fueron trabajados en forma dicotómica, no sin antes explorar los resultados previos a las recodificaciones. Dado el reducido número de casos, el objetivo de este 
análisis estadístico fue esencialmente exploratorio. En una segunda fase de análisis se produjeron grafos de todos los casos con el software Ucinet 6, permitiendo la exploración visual.

\section{Principales hallazgos del estudio de redes personales}

El punto de partida del estudio de redes personales se encontraba en la validación, a partir de los resultados de la encuesta general, de la hipótesis sobre el impacto de Internet en el patrón de relaciones de sus usuarios frecuentes en las comunas consideradas al expandir los vínculos tanto en términos cantidad como de heterogeneidad. Ahora bien, tal y como se pudo comprobar posteriormente a través de los focus group, cuanto mayor es el nivel de aislamiento mayor relevancia toma la presencialidad para poder valorizar este vínculo como un medio que realmente signifique acceso a recursos. En otras palabras, al comparar los datos de los encuestados en la región de Aysén con aquellos procedentes de los casos de las comunas de la Región Metropolitana, con un nivel de aislamiento menor, se evidencia en los primeros una menor predisposición a conocer gente nueva por Internet. Pero además, este tipo nuevas relaciones mediadas por lo virtual no son percibidas como una fuente potencial de apoyo significativo, discurriendo por vías paralelas a las relaciones presenciales o semi-presenciales. Lo que vendría a avalar lo planteado por otros autores con respecto que a pesar de la instantaneidad temporal y cobertura espacial que permite en Internet en la construcción de relaciones, el peso de factores socioculturales propio de las zonas rurales y aisladas siguen pauteado la construcción del vínculo (Proenza, 2002; Cárcamo y Cladellas, 2009).

Una derivada en el trabajo analítico a partir de esta primera evidencia nos lleva, conjuntamente con los datos de la encuesta general, a desarrollar un análisis de conglomerados para identificar tres tipos de perfiles relacionales exclusivamente entre los casos de los encuestados en la región de Aysen. El resultado son tres perfiles claramente identificados. Un primer conglomerado, el "A", muestra a sujetos aislados o descapitalizados en términos digitales, ya que no le dan ningún uso social a Internet. Por este motivo son los que conocen un menor número medio de personas $\mathrm{y}$, además, cuentan con escasas fuentes a las cuales acudir en caso de necesitar ayuda. Por el contrario, el segundo conglomerado, el "B", nos habla sobre los sujetos más activos en la red, el principal uso que dan a Internet está orientado a conocer personas nuevas, y algunos de ellos, aunque son los menos, también usan la red para contactarse con sus amistades y conocidos. En esta línea, son los que, por lejos, conocen una mayor cantidad de personas a través de la red; y también cuentan con un mayor número de fuentes de capital social. Por este motivo se ha denomina a estos encuestados como capitalizados digitales exogámicos. Por último, el tercer conglomerado nos habla de sujetos que recrean los lazos que ya poseen, y se dedican nada o poco a conocer nuevas personas. La media de personas nuevas conocidas a través de Internet es bastante menor respecto de los sujetos del conglomerado " $\mathrm{B}$ ”, aunque mayor respecto de los del conglomerado " $A$ ”, pero se asemeja a este último al compartir un bajo número de fuentes 
de capital social. Así entendido, se ha decidido definir el perfil de los entrevistados del conglomerado B como sujetos capitalizados digitales endogámicos.

Tabla 3. Definición de los conglomerados

\begin{tabular}{|c|c|c|c|c|c|}
\hline Conglomerado & $\begin{array}{c}\text { Uso de } \\
\text { Internet } \\
\text { para } \\
\text { conocer } \\
\text { personas } \\
\text { nuevas (1) }\end{array}$ & $\begin{array}{c}\text { Uso de } \\
\text { Intemet para } \\
\text { relacionarse } \\
\text { con } \\
\text { amistades (2) }\end{array}$ & $\begin{array}{c}\text { Número } \\
\text { medio de } \\
\text { personas } \\
\text { conocidas } \\
\text { por } \\
\text { Internet(2) }\end{array}$ & $\begin{array}{c}\text { Número } \\
\text { medio de } \\
\text { fuentes de } \\
\text { capital } \\
\text { social (3) }\end{array}$ & $\begin{array}{c}\text { Tamaño del } \\
\text { conglomerado } \\
\%(N)\end{array}$ \\
\hline A & Nunca & Nunca & 1,48 & 1,08 & $36,7 \%(165)$ \\
\hline B & Siempre & $\begin{array}{c}\text { Nunca y } \\
\text { Siempre }\end{array}$ & 21,72 & 1,32 & $32,3 \%(145)$ \\
\hline C & Nunca & Siempre & 3,17 & 1,26 & $31,0 \%(139)$ \\
\hline
\end{tabular}

Relevancia de la variable en la formación del conglomerado: (1) Alta; (2) Media; (3) Baja.

Estos hallazgos aportados por la encuesta general pasaron a ser abordados, posteriormente, a través del estudio de redes personales para profundizar en su conocimiento a través de este método. El primer punto a considerar al momento de caracterizar la red social de apoyo de nuestros encuestados era su tamaño. Al respecto, la cifra promedio de alteri referidos era de 11,8. Una cifra que prácticamente no varía la cruzar la variable sobre frecuencia en el uso de Internet. Las diferencias se plantean al momento de aproximarnos a la estructura de la red.

En promedio, el número de relaciones reconocidas entre los alteri mencionados por los encuestados es, para los usuarios con alta frecuencia de Internet, de 83,4, mientras que para los usuarios con baja frecuencia de 72,4 . Al proyectar estos datos en términos de densidad o porcentaje de relaciones existentes con respecto a las potenciales, los resultados son muy similares: 66\% para los encuestados con alta frecuencia de uso de Internet y 68\% para usuarios con baja frecuencia. En ambos casos, se trata de porcentajes muy altos, siendo reflejo de la pertenencia a estructuras sociales compactas propio de la pertenencia a comunidades locales.

Ahora bien, al aproximarnos a la configuración de las redes personales en razón del lugar de residencia de los alteri, empiezan a emerger algunas diferencias relevantes para el propósito de nuestro estudio. Tal y como se muestra en la tabla n.4, si bien para la mayoría de los encuestados las relaciones se encuentran insertas en el medio local, las personas que utilizan Internet en forma más frecuente tienen mayor número de vínculos fuera de este ámbito que los que no comparten esta condición. 
Polis, Revista Latinoamericana, Volumen 13, $N^{\circ}$ 38, 2014

\section{Tabla 4. Distribución de vínculos según localización}

\begin{tabular}{|c|c|c|}
\hline & Vínculos Internos & Vínculos Externos \\
\hline Baja frecuencia uso Internet & $83,3 \%$ & $16,7 \%$ \\
\hline Alta frecuencia uso Internet & $71,4 \%$ & $28,6 \%$ \\
\hline
\end{tabular}

Los grafos que se presentan a continuación corresponden a la representación visual de las redes personales de dos de los encuestados de una misma localidad, Cerro Castillo, pero con condición de uso de Internet diferentes. El grafo n.1 corresponde a María José, estudiante de 17 años con uso diario y constante de Internet. Identificó 22 alteri como potenciales fuentes de apoyo: 8 corresponden a la categoría de familiares y 14 a las de amigos. En cuanto la residencia de los contactos, 8 residen también en Cerro Castillo (identificados en el grafo en gris) y el resto, 14, en diferentes localidades de la región de Aysén (color negro). Con estos últimos se mantiene una relación constante o regular a través de Internet, pero en cualquier caso el inicio del proceso de construcción del vínculo se inició presencialmente. El tipo de apoyo esperado por parte de aquellos alteri de Cerro Castillo es, en la mayoría de los casos, diferente al que se espera en aquellos que se encuentran fuera de la localidad de residencia. Mientras que a los primeros se les percibe como fuente de apoyo ante posibles problemas que afectan a la condición presente, como problemas económicos o de salud, a la mayor parte de los alteri externo se les identifica como fuente de apoyo en términos de mejora de la condición presente. Lo que involucra, por ejemplo, obtención de información sobre oportunidades de estudio, laborales o de ocio.

\section{Grafo n.1. Caso Red Personal de María José}

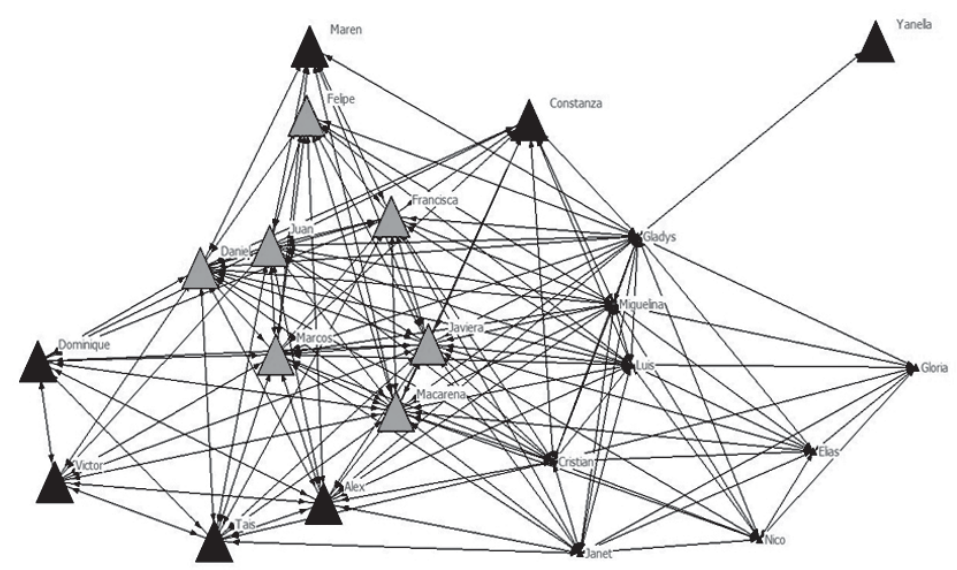

Fuente: Elaboración propia con aplicación de software Ucinet. 
El primer dato a destacar al momento de caracterizar la estructura de red conformada por las vinculaciones entre los alteri de Maria José es que la densidad se sitúa alrededor del 60\%. Ahora bien, se trata de una densidad que se distribuye de forma desigual al interior de la red. La densidad más alta, como cabria de esperar, se concentra entre los alteri que comparten residencia en Cerro Castillo, quiénes comparten una vinculación directa entre todos ellos y a su vez conexiones con los alteri que residen en otras localidades. Principalmente entre aquellos con los que mantienen un vínculo familiar. La situación es diferente en el caso de los alteri externo. Nuevamente la condición de familia favorece una mayor conectividad, pero en los amigos la densidad varía. La aplicación de la técnica de los cliques permite aportar más luz sobre esta propiedad de la red al poner de relieve no sólo que la mayor parte de triadas o cliques conformado por tres actores se producen entre los alteri residentes en la misma localidad, sino que éstos se reproducen de forma consistente solamente con algunos actores externos.

Bajo estas circunstancias, al aplicar un análisis de los principales indicadores de centralización de las distintas redes sociales (grado, intermediación y cercanía) los resultados dan cuenta de una red en la que los puntajes son altos o mayoritariamente altos. Por otro lado, como puede comprobarse en la tabla n.5, que son muy similares a los resultados promedios de los cálculos de centralidad en el caso del conjunto de encuestados bajo la condición de usuarios frecuentes de Internet. Se trata, por tanto, en el que tienen cierto peso los vínculos verticales que restringen la cohesión. Lo que debe interpretarse como una red que presenta un capital social brinding o de puente al permitir la circulación de recursos entre personas con atributos sociodemográficos relativamente distintos.

Tabla 5. Cálculo de Centralidades

\begin{tabular}{|l|c|c|c|}
\hline & $\begin{array}{l}\text { Centralidad } \\
\text { de Grado }\end{array}$ & $\begin{array}{l}\text { Centralidad } \\
\text { de Cercanía }\end{array}$ & $\begin{array}{l}\text { Centralidad } \\
\text { de Intermediación }\end{array}$ \\
\hline Caso Estudio María José & 0,34 & 0,40 & 0,12 \\
\hline $\begin{array}{l}\text { Promedio General Usuarios } \\
\text { Frecuentes de Internet }\end{array}$ & 0,34 & 0,39 & 0,17 \\
\hline
\end{tabular}

El grafo n.2 corresponde a Gabriela, residente también en Cerro Castillo y que manifiesta conectarse a Internet "no más allá de una vez por semana”. Se trata de una dueña de casa de 31 años que reconoció 10 alteri como principales fuentes de apoyo, 6 de los cuáles son familiares y 4 amigos. De éstos la mitad residen en Cerro Castillo (gris) y el resto entre Coyhaique y Puerto Aysén (negro). El contacto con los primeros se establece a través de encuentros presenciales, mientras que con los segundos se combina el teléfono, el Internet y, de forma muy esporádica, la cita presencial. El apoyo esperado en todos los casos es el mismo, recursos que le permitan solventar eventuales problemas económicos o de salud en su familia. 


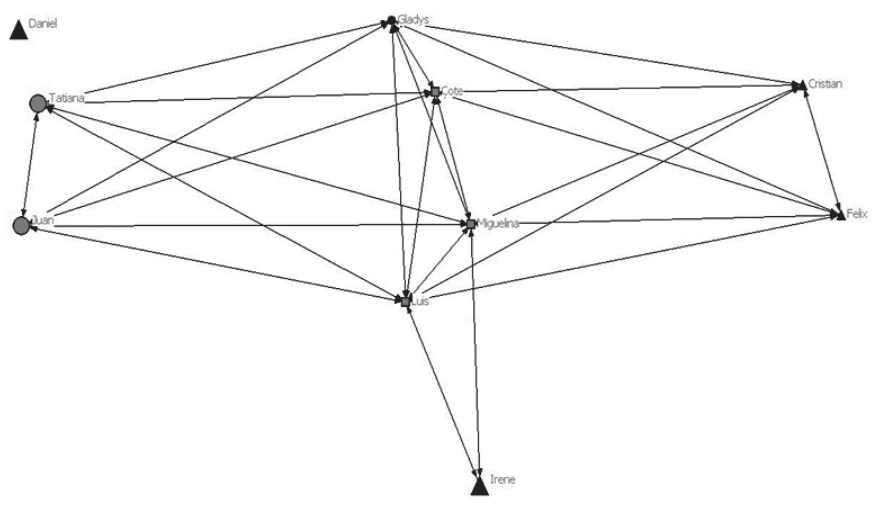

La densidad de la red de Gabriela es cercana al 65\%. Ahora bien, se trata de un dato muy sesgado por la presencia de un nodo aislado dentro de la red, Daniel. Al momento de dejar fuera del cálculo este nodo la densidad en las relaciones entre el resto de miembro de la red de Gabriela aumenta a casi el 82\%. Lo que refleja una estructura de relaciones muy compacta en la que la mayoría de actores se vinculan directamente entre ellos. Principalmente entre los que residen en la misma localidad, pero también entre los escasos actores externos excepto el caso señalado anteriormente. Como consecuencia de esta situación, el cálculo de las medidas de centralidad de la red da cuenta de puntajes bajos o mayoritariamente bajos. Se trata, por tanto, de una red conformada por vínculos mayoritariamente horizontales y cohesionadas a través de la que se canalizan recursos entre personas relativamente similares. En otras palabras, lo que en la literatura se identifica como capital social bonding o de nexo.

Tabla 6. Cálculo de Centralidades

\begin{tabular}{|l|c|c|c|}
\hline & $\begin{array}{l}\text { Centralidad } \\
\text { de Grado }\end{array}$ & $\begin{array}{l}\text { Centralidad } \\
\text { de Cercanía }\end{array}$ & $\begin{array}{l}\text { Centralidad } \\
\text { de Intermediación }\end{array}$ \\
\hline Caso Estudio Gabriela & 0,22 & 0,26 & 0,09 \\
\hline $\begin{array}{l}\text { Promedio Usuarios de Baja } \\
\text { Frecuencia de Internet }\end{array}$ & 0,26 & 0,29 & 0,12 \\
\hline
\end{tabular}

\section{Conclusiones}

¿Cuál es el valor que aporta el método del estudio de redes personales? En la línea de lo planteado por otro autores (Ferrand, 2002; de Federico, 2002; Molina, 2005), nuestra respuesta ahonda en su contribución a la explicación de fenómenos sociales de rango "intermedio" o meso. Es decir, aquellos fenómenos susceptibles de ser explicados a partir de la interacción 
entre individuos, organizaciones, instituciones y estructuras sociales de forma simultánea. Desde la perspectiva del pensamiento estructural, el desafío que se plantea es la identificación, ordenación y articulación sistemática de estas interacciones observables empíricamente. Bajo el supuesto que el medio social puede ser explicado como pauta de regularidades en las relaciones entre unidades en interacción (Wasserman y Faust, 1994), no puede dudarse que el ARS ofrece a las Ciencias Sociales en su conjunto y, en particular, a la sociología una propuesta metodológica con gran potencial para responder a preguntas que difícilmente pueden ser explicadas desde las aproximaciones individualistas.

Retomando el marco temático en que hemos situado nuestro artículo, la contigüidad espacial de la concepción de la comunidad la alternativa metodológica propuesta nos conduce a conocer la estructura dual de relaciones sociales que tienen los individuos en razón, principalmente, de su localización geográfica y su condición de usuario de Internet. Esta dualidad puede ser formulada en términos estructurales a partir del concepto de puente. La ventaja de esta posición se encuentra en la capacidad de conectar dos sub-redes diferentes y no relacionadas (Ferrand, 2002). Uno de los objetivos principales de la investigación presentada fue analizar cómo el uso frecuente de Internet permitía capitalizar de forma diferenciada, interna y externamente, la suma de nuevos vínculos. Pero a su vez comprender si esta modificación en el capital social individual tenía algún impacto real en el capital social colectivo dentro de un contexto local especialmente favorable, dado su aislamiento geográfico, por su clausura relacional. La aplicación del método del estudio de redes personales, a partir de la base establecida por la encuesta general y los datos aportados por los otros instrumentos, permitió explorar la potencialidad de esta eventual transferencia y plantear tentativamente la emergencia de la figurar del identificado como puente interlocal digital. 
Polis, Revista Latinoamericana, Volumen 13, $N^{\circ}$ 38, 2014

\section{Notas}

${ }^{1}$ Este artículo es parte del Proyecto FONDECYT (n. 1120866) El Impacto de Internet en el Capital Social de las Comunidades Aisladas. Una versión preliminar de este documento fue presentado en un workshop realizado durante mi estadía como investigador visitante en el IN3, Universitat Oberta de Catalunya, Barcelona. Agradezco el apoyo brindado por Maureen Lozier y Andres Keller en el procesamiento de datos.

${ }^{2}$ La idea del Mundo Pequeño tiene su origen en varios experimentos llevados a cabo por el psicólogo social Stanley Milgram en Estados Unidos. Lo innovador de esta investigación, fue la revelación de que la sociedad humana es una red social que presenta la estructura del mundo pequeño, caracterizada por interconexiones mucho más cortas de lo esperadas.

${ }^{3}$ Véase al respect Silvia Domínguez y Bettina Hollstein (2014) Mixed Methods Social Networks Research, Cambridge University Press.

${ }^{4}$ El programa Egonet (Personal Network Software) está orientado al análisis de redes personales (egonetworks), que son un conjunto de personas (alters) que tienen alguna relación operacionalizada en términos del estudio con una persona central (ego). Egonet es un programa diseñado para la recolección de datos y análisis de redes egocéntricas. Su creador es el profesor Christopher McCarthy, de la Universidad de la Florida, USA.

${ }^{5}$ Una completa información sobre los objetivos, las actividades y principales hallazgos del proyecto puede obtenerse visitando la web: www.internetycapitalsocial.cl 


\section{Bibliografía}

Antoci, A., Sabatini, F., y Sodini, M. (2012), "See you on Facebook! A framework for analyzing the role of computer-mediated interaction in the evolution of social capital", en The Journal of Socio-Economics, 41, 541-547.

Barabási, A. (2002), Linked. The New Science of Networks, MA, Perseus Publishing, Cambridge.

Bauernschuster, S., Falck, O., y Woessmann, B. F. (2011), "Surfingalone? The Internet and social capital: Evidence from an unforeseeable technological mistake". IZA, Discussion Paper Serie N5747.

Bauman, Z. (2003), Modernidad líquida, FCE, Argentina.

Ídem (2004), La comunidad, Siglo XXI, Madrid.

Beaudoin, C. E. (2008), "Explaining the relationship between Internet use and interpersonal trust?: Taking into account motivation and information overload", en Journal of Computer-Mediated Communication, 13, 550 568.

Bell, D.(2001), The Future of Technology, Cambridge University Press, Mass.

Burke, M., Kraut, R., y Marlow, C. (2011), " Social capital on Facebook?: Differentiating uses and users", en ACM CHI 2011: Conference on Human Factors in Computing Systems.

Cárcamo, L. y R. Cladellas (2009), "Contextos culturales y percepción del tiempo en Chile", en Revista Austral de Ciencias Sociales, 17, 99-108.

Cohen, A. P. (1985), The symbolic construction of community, Londres, Routledge.

Ellison, N. B., Steinfield, C., y Lampe, C. (2007), "The benefits of Facebook 'friends': Social capital and college students' use of online social network sites", en Journal of Computer-Mediated Communication, 12, 1143-1168.

Festinger, L., Schacter, S., Back, K. (1950), Social pressures in informal groups: A study of human factors in housing, Calif., Stanford University Press, Stanford.

Fischer, C. S. (1982), To dwell among friends: personal networks in town and city, The University of Chicago Press, Chicago.

Ídem (1994), America Calling: A social history of the telephone to 1940, University of California Press, Berkeley. 
Giddens, A (1991), Modernity and Self-Identity, Stanford University Press: CA

Hillery, G. (1955), “Definitions of community: areas of agreement”, en Rural Sociology, 20, 111-123.

Kadushin, C. (2013), Understanding Social Networks: theories, concepts, and findings, Oxford University Press, Oxford.

Knoke, D., Kuklinski, J. (1982), Network Analysis, CA, Sage, 1982, Beverly Hills.

Kraut, R., Kiesler, S., Boneva, B., Cummings, J., Helgeson, V., y Crawford, A. (2002), "Internet Paradox Revisited", en Journal of Social Issues, 58(1), 49-74.

Lazarsfeld, P., Merton, R. K. (1955). "Freedom and control in modern society”, en Friendship as a social process: A substantive and methodological analysis. Octagon Books, New York.

Lee, D., Newby, H. (1983), The problem of sociology: an introduction to the discipline, Unwin Hyman, Londres.

Lin, N. (2001), Social capital: A theory of social structure and action, Cambridge University Press, Cambridge.

McCarthy, C. (2010), “La estructura en las redes personales” en Revista Redes, Vol.19,\#11, Diciembre 2010

McPherson, M., Sith-Lovin, L., Cook, J. M. (2001). "Birds of a feather: Homophily in social networks", en Annual Review of Sociology, 27, 415444.

Mejias, J. (2000), “El muestreo en la investigación cualitativa” en Revista Peruana de Investigaciones Sociales, Universidad de San Marcos, Lima.

Miyata, K., y Kobayashi, T. (2008), “Causal relationship between Internet use and social capital in Japan”, en Asian Journal of Social Psychology, 11, 42-52.

Molina, J.L. (2004), “La ciencia de las redes”, Apuntes de Ciencia y Tecnología 11, Junio (36-42)

Molina, J. L. (2005), “El estudio de las redes personales: contribuciones, métodos y perspectivas”, en Empiria - Revista de Metodología de Ciencias Sociales, 10 (julio-diciembre), 71-105.

Pénard, T., y Poussing, N. (2010), "Internet Use and Social Capital?: The Strength of Virtual Ties”, en Journal of Economic Issues, 44(3), 569-595. 
PNUD (2000), Desarrollo Humano en Chile. PNUD, Santiago.

Poplin, D. E. (1972), Communities: a survey of theories and methods of research, Macmillan, NuevaYork.

Proenza, F. et al (2002), Telecentros para el desarrollo socioeconómico y rural en América Latina, BID, Washington.

Putnam, R. D. (1996), “The strange disappearance of civic America”, en American Prospect, 7(24). Retrieved November 1st, 2005, from TAP Website: http://www.prospect.org/print/V7/24/putnam-r.html.

Ídem (2002), Solo en la bolera: Colapso y resurgimiento de la comunidad norteamericana, Galaxia Gutenberg, Barcelona.

Ídem (1995), Bowling alone. America`s decline of social capital.

Sampson, R. J. (1999), “What “community” supplies”, en Ferguson, R. F., Dickens, W. T. (eds.), Urban problems and community development, D.C., Brookings Institution Press, Washington, 241-279.

Sennett, R. (2001), Vida urbana e identidad personal, Ediciones Península, Barcelona.

Scott, John (2000), Social Network Analysis, Sage, Londres.

Sum, S., Mathews, M., Pourghasem, M., y Hughes, I. (2008), “Internet Technology and Social Capital?: How the Internet Affects Seniors' Social Capital and Wellbeing”, en Journal of Asian and African Studies, 14, 202-220.

Tavvsko García, R (2013), Cuestiones sobre identidad y nación vasca en internet, Deusto, Bilbao

Turkle, S, (1995), Life on the Screen: Identity in the Age of the Internet, Touchstone

Valenzuela, S., Park, N., y Kee, K. (2009), “Is there social capital in a social network site?: Facebook use and college students ' life satisfaction, trust, and participation", en Journal of Computer-Mediated Communication, 14, 875-901.

Verburgge, L. (1977), “The structure of adult friendship choices”, en Social Forces 56, 576-597.

Wasserman, S., Galaskiewicz, J. (eds.) (1994), Advances in Social Network Analysis, Sage, Thousand Oaks, CA.

Watts, D. J. (1999), Small Worlds. The Dynamics of Networks between Order and Randomness, Princenton University Press, Princenton. 
Polis, Revista Latinoamericana, Volumen 13, $N^{\circ}$ 38, 2014

Wellman, B. (1992), “Domestic affairs and network relations”, en Journal of Social and Personal Relationships, 9, 385-409.

Ídem (1998), “The network community. An introduction”, en Wellman, B. (ed.). Networks in the global village: life in contemporary communities, Westview Press, 1-48, Boulder (CO).

Ídem (2001), "Physical place and cyberplace: the rise of personalized networking”. International Journal of Urban and Regional Research 25(2), 227-52.

Recibido: 24.06.14

Aceptado: 10.12.2014 